\title{
Management of Mandibular Fractures
}

\author{
Col GK Thapliyal", Col R Sinha ${ }^{+}$, Col PS Menon", Surg Lt Cdr A Chakranarayan**
}

\begin{abstract}
Background: To evaluate the cases of mandibular fractures treated by open reduction and rigid fixation at the Armed Forces Medical College, Pune.

Methods: Two hundred and thirteen mandibular fractures cases were treated by open reduction and rigid fixation from Jun 1998 to Jun 2006. These were retrospectively analyzed based on the following patient related factors i.e. mode of injury, age and sex distribution, site of injury, associated injuries and surgical treatment.

Result: The significant finding was that the most common etiology for fracture mandible was road traffic accidents (RTA), [196 $(92 \%)]$. Of the patients who reported, $147(95.4 \%)$ were males in the third decade of life. $96(45 \%)$ patients had parasymphysis fractures, $65(30.5 \%)$ had angle fractures, $33(15 \%)$ subcondylar fractures, $17(7.9 \%)$ body fractures and $2(0.9 \%)$ had ramus fracture. Early intervention using open reduction and internal fixation was the protocol followed which resulted in minimum morbidity and complications.

Conclusion: As RTA especially two wheelers accidents appeared to be the most common cause of mandibular fractures, use of suitably designed protective head gear (crash helmets) is advisable. Moreover open reduction and rigid internal fixation appears to be the suitable treatment modality in successful treatment of mandibular fractures in reducing morbidity and complications and ensuring early return to normalcy.
\end{abstract}

MJAFI 2008; 64 : 218-220

Key Words : Mandibular fracture; Road traffic accident; Parasymphysis

\section{Introduction}

$\mathrm{T}$ The increasing number of vehicles and the deleterious condition of roads has led to a significant increase in craniofacial trauma. Mandible fractures occupy the second most frequent incidence of facial bone fractures, with incidence of about $38 \%[1,2]$. These are mainly caused by two-wheeler accidents. The mandible is a resistant bone and it takes a relatively heavy impact to fracture, which apart from road traffic accidents (RTA) can also be a consequence of sport activities, firearms, interpersonal violence, work-related accident and pathological conditions. Since the mandible is the mobile facial bone, a fracture is generally never left unnoticed because it is very painful, worsening with mastication and speech movements and if left untreated may cause facial asymmetry. Mandible fractures may lead to deformities, either due to displacement of the fracture fragments or non-restored bone losses, with a disturbed dental occlusion with or without temporomandibular joint disorders. Consequences of an untreated or inappropriately treated mandibular fractures may be severe, both cosmetically and functionally. The present study was aimed at assessing 213 patients who were treated for mandible fractures at Armed Forces Medical
College, Pune, according to gender, age, etiology, location of fractures, treatment techniques and postoperative complications.

\section{Material and Methods \\ Patients reporting to the out patient department or casualty} with maxillofacial trauma or transferred from peripheral service hospitals with a diagnosis of mandibular fractures from June 1998 to June 2006 were treated by open reduction and internal fixation. We performed an observational epidemiological, descriptive and retrospective study of the medical records of these cases. During the initial period of five years, record keeping was restricted to radiographs with details of the patient and operative procedures recorded in the treatment book. Since 2004, complete digital records of pre operative, operative and postoperative clinical photographs with pre operative and postoperative radiological records are available. The average time from reporting to surgical intervention was five days.

The diagnosis was based on clinical and radiological examination. The standard radiographs used were (i) Posterioanterior view mandible (ii) Reverse Towne's view mandible (iii) Lateral oblique mandible (iv) Orthopantomogram (OPT) (v) Intraoral periapical (IOPA) radiographs, depending on the site and extent of the fractures. All cases were treated under general anaesthesia by rigid internal fixation following

*Senior Specialist (Oral \& Maxillofacial Surgery), AFDC, New Delhi. ${ }^{+}$Professor \& Head, *Associate Professor (Oral \& Maxillofacial Surgery), ${ }^{* *}$ Resident (Oral \& Maxillofacial Surgery), Department of Dental Surgery, AFMC, Pune-40.

Received : 05.04.07; Accepted : 17.12.07_Email: thapliyalgk@rediffmail.com 
maxillomandibular fixation (MMF) using an arch bar or Intermaxillary fixation (IMF) screws. The sequence of rigid fixation was distal to proximal, tackling the dentoalveolar fractures first. The surgical approaches used to access the fracture site were transoral for parasymphysis, body and angle fractures. For the low subcondylar and high subcondylar fractures, the postramal and the preauricular approaches were used respectively. MMF with elastics was done in the preoperative and initial postoperative period. MMF was maintained for a period of five to seven days following which it was removed once desired dental occlusion was maintained.

\section{Results}

In the last six years there has been no significant change in etiological factors, with RTA being the major contributory factor. The quantum of fractures has shown an insignificant increase with respect to craniofacial region including the mandible. There was a significant rise in the number of cases in the monsoon and the post monsoon period. Mandibular fractures comprised of $45.5 \%$ of the total cases reporting with maxillofacial trauma. $11.73 \%$ cases had associated injuries of the maxillofacial region, of which zygomatic complex fractures was $6.1 \%$, dentoalveolar fractures $2.3 \%$, Lefort I fractures $2.8 \%$ and Lefort II $0.4 \%$. RTA-196 (92\%) was the most common etiological factor followed by fall - 7 (3.28\%), interpersonal violence $-5(2.3 \%)$, sports injuries $-3(1.4 \%)$ and gun shot wounds (GSW) -2 (1.1\%).

Age distribution with decreasing incidence rates were : third decade (45.5\%), fourth decade (29.4\%), second decade (14.7\%), fifth decade (6.6\%), sixth decade (2.2\%), seventh decade (1.1\%) first decade $(0.7 \%)$ and eighth decade $(0.7 \%)$ out of which $95.5 \%$ were males and $4.4 \%$ females. Site distribution was : 96 (45\%) parasymphysis, 65 (30\%) angle, $33(15 \%)$ condylar, $17(7.9 \%)$ body and $2(0.9 \%)$ ramus, of which $122(57 \%)$ were single mandibular fractures and 91 (43\%) were cases with multiple mandibular fractures.

The average time from injury to surgical intervention was five days. All cases were treated by open reduction and internal fixation using miniplate osteosynthesis except one case in which wire osteosynthesis was used. The clinical presentation of patients when they first arrived at AFMC comprised of specific signs and symptoms of trauma (pain and local edema), with specific features of mandible fracture (difficulty to open the mouth, deviation on opening the mouth, malocclusion). Complete workup of the patients was done which included a detailed history and hematological and urine examination. Radiological investigation was based on the site of injury and the presenting clinical features. Computed tomography (CT) scans were performed in cases of concomitant head injury and / or pan facial trauma cases. For patients above 35 years of age a chest radiograph, ECG and blood sugar (fasting / postprandial) was done as a part of preanesthetic checkup protocol. All patients were given a loading dose of antibiotics and steroids intravenously prior to surgery. The surgical protocol followed for fracture fixation was based on Champy's lines of osteosynthesis. Miniplates were used in all cases for rigid internal fixation. Two miniplates one at the lower border and one below the root apices were used for the parasymphysis region fractures, one plate at the surgical angle for the angle fractures and one or two plates for the subcondylar fractures parallel to the posterior border of the ramus. Postoperatively antibiotics and antiinflammatory medication were given for three to five days. Steroids were stopped on the second postoperative day. The patients were allowed soft diet from the first postoperative evening. Intraoral and facial skin sutures were removed after seven days and five days respectively. Radiographs were obtained postoperatively prior to discharge and after six weeks. The most common complication resulting from the treatment of mandible fracture was minor occlusal discrepancy during the early postoperative period. This was managed successfully by MMF using elastics in the postoperative period. 14 of the $213(6.57 \%)$ cases treated with mandibular fractures had persistent occlusal discrepancies that persisted in the postoperative phase. These discrepancies were essentially the inability of the patient to interdigitate the maxillary and mandibular teeth in maximum intercuspation. Most of these were seen in subcondylar fractures where the surgical procedure results in inflammation of the temporomandibular joint resulting in this problem. Guiding elastics and regression of inflammation helps resolve this problem.

The other postoperative complication we faced were infections at the site of rigid fixation in 21 cases, which were successfully managed by surgical removal of the implant after the healing phase.

\section{Discussion}

Although being the heaviest and strongest facial bone, the mandible is prone to fractures because of the anatomical peculiarity of form and location. There has been an increase in number of traffic accidents and interpersonal violence contributing to an increase in the frequency of mandibular fracture [1-4]. The age range of 20 to 29 years was the most affected $[5,6]$. The male predominance in the age range 20-29 years is due to the increased use of two wheelers by this group and also the prevalent interpersonal violence, normally associated with use of alcoholic beverages. This gender bias observed in our study is in agreement with the existing literature [7]. The causes of fracture were extremely variable. In the present study, the decreasing order of frequency found was: road traffic accidents, interpersonal violence, sport, GSW, and other factors. There are countries where the main cause of mandible fracture is related with sport activities, such as in Austria [8]. Others present interpersonal violence as the most common cause, such as Hawaii, Zimbabwe and Canada $[9,10,11]$. We classically find a set of signs and symptoms comprising of pain, edema, hematoma, deranged dental occlusion, facial contour deficit, grating and mobility of bone fragments. The radiological investigations range from anteroposterior/ posteroanterior view mandible, $30^{\circ}$ right and left oblique view lateral mandible, OPT, 
occlusal view to intraoral periapical view.

Most mandibular fractures in the present study occurred in isolation. Although the total incidence of associated maxillofacial fractures was lower than that reported in most studies, zygomatic complex was the most common associated site. Out of the nearly 25 cases that had associated injuries of the maxillofacial region with concomitant mandibular fractures, $90 \%$ of these were encountered in the last three years. This could be due to the increased speeds of vehicles and increased density of two wheelers in the last few years. The mandible fracture site is variable, depending on the many different causes of the fracture. What was significant is that there was a gradual change in incidence of mandibular fractures from single fractures to multiple areas of fracture over this period. Of the multiple regions affected, 63 of the 122 involved the parasymphysis and opposite angle, 17 involved parasymphysis and opposite subcondylar region, 15 were bilateral angle fractures, nine were bilateral parasymphysis fractures, seven were body and parasymphysis fractures, rest had an added component of dentoalveolar fractures. Literature differs a lot concerning the affected sites. In the present study, the parasymphysis and mandibular angle were the most affected. There are many different therapeutic possibilities, given that many authors disagree about the best treatment approach $[12,13]$. The objective of therapy includes absence of pain, satisfactory dental occlusion, maximum interincisal opening $(35 \mathrm{~mm})$ and mandibular / facial symmetry. At our institution mandible fractures were treated with two basic forms of treatment, either open or closed approach. Closed treatment was used in children. Open treatment has been the protocol for all mandibular fractures in adults at our institution. The surgery, which is based on the principle of reduction and fixation of bone fragments, should be conducted as soon as clinical conditions allow. Most patients were discharged after seven to ten days, confirming the low rate of postoperative complications.

In the present study, there was a predominance of males in the second and third decade of life reporting with maxillofacial injury consisting of mandibular fractures. The most common cause was road traffic accident and the more frequently affected region on the mandible was the parasymphysis and angle respectively. Most patients were treated by open reduction within the first five days and were discharged after the early postoperative period. The most frequent complication encountered was that of transient malocclusion.

\section{Conflicts of Interest}

None identified

Intellectual Contribution of Authors

Study Concept : Col R Sinha

Drafting \& Manuscript Revision : Col P S Menon

Statistical Analysis: Surg Lt Cdr A Chakranarayan

Study Supervision : Col GK Thapliyal, Col PS Menon

\section{References}

1. Krishnaraj S, Chinnasamy R. A 4-year retrospective study of mandibular fractures in a South Indian city. J Craniofac Surg. 2007; 18:776-80.

2. Subhashraj K, Nandakumar N, Ravindran C. Review of maxillofacial injuries in Chennai, India: A study of 2748 cases. Br J Oral Maxillofac Surg 2007; 22: 298-302.

3. Sojat AJ, Meisami T, Sandor GKB, Clokie CML. The epidemiology of mandibular fractures treated at the Toronto General Hospital. A review of 246 cases. J Can Dent Assoc 2001; 67:640-4.

4. Schön R, Roveda SIL, Carter B. Mandibular fractures in Townsville, Australia: incidence, etiology and treatment using the 2.0 AO/ASIF miniplate system. British Journal of Oral and Maxillofacial Surgery 2001; 39: 145-8.

5. El-Degwi A, Mathog RH. Mandibular fractures - Medical and economic considerations. Otolaryngol Head Neck Surg 1998; 108: 213-9.

6. Simsek S, Simsek B, Abubaker AO, Laskin DM. A comparative study of mandibular fractures in the United States and Turkey. International Journal of Oral and Maxillofacial Surgery 2007; 36:395-7.

7. Nicholas Z, Michael M. Fractures of the mandibular condyle: A review of 466 cases. Literature review, reflections on treatment and proposals. Journal of Cranio-Maxillofacial Surgery 2006; 34:421-32.

8. Schoning R, Rothler H, Waldhart G. Trends in the incidence and cause of sport-related mandibular fractures: a retrospective analysis. J Oral Maxillofac Surg 1997; 55: 585-92.

9. Bach TL, Eric JD, Brett AU, Louis DH, Bryce FP. Maxillofacial injuries associated with domestic violence. Journal of Oral and Maxillofacial Surgery 2001;59: 1277-83.

10. Ogundare BA, Bonnick A, Bayley N. Pattern of mandibular fracture in urban major trauma centre. J Oral Maxillofac Surg $2003 ; 61: 713-8$.

11. Motamedi MH. An assessment of maxillofacial fractures. A 5year study of 237 patients. J Oral Maxillofac Surg 2003; 61: $61-4$.

12. Andersson J, Hallmer F, Eriksson L. Unilateral mandibular condylar fractures: A 31-year follow-up of non-surgical treatment. Int J Oral Maxillofac Surg 2007; 36:310-4.

13. Eckelt U, Schneider M, Erasmus F, Gerlach KL, Kuhlisch E, Loukota R, et al. Open versus closed treatment of fractures of the mandibular condylar process-a prospective randomized multi-centre study. J Craniomaxillofac Surg 2006; 34:306-14. 